\title{
A Rare Case of Autosomal Dominant Polycystic Kidney Disease Presenting in utero
}

\author{
${ }^{1}$ Yeshita Pujar, ${ }^{2}$ Babasaheb Raosaheb Desai, ${ }^{1}$ B havana Sherigar, ${ }^{1}$ Hema Dhumale, ${ }^{1}$ Geeta Durdi, ${ }^{1}$ Anita Dalal \\ ${ }^{1}$ Associate Professor, Department of Obstetrics and Gynecology, J awaharlal Nehru Medical College, Belgaum, Karnataka, India \\ ${ }^{2}$ Professor, Department of O bstetrics and Gynecology, J awaharlal Nehru Medical College, Belgaum, Karnataka, India
}

Correspondence: Yeshita Pujar, Associate Professor, Department of Obstetrics and Gynecology, J awaharlal Nehru Medical College, Nehru Nagar, B elgaum-590010, Karnataka, India, Phone: 09448142989, Fax: 0831-2470759, e-mail: yvpujar@ hotmail.com

\section{ABSTRACT}

Autosomal dominant polycystic kidney disease (ADPKD) is the most widespread cause of genetic nephropathy. Only $25 \%$ of patients are symptomatic. ${ }^{1}$ One in 1,000 people carries the autosomal dominant polycystic kidney disease mutant gene. Autosomal dominant polycystic kidney disease is usually asymptomatic until the third or fourth decade of life, although histological evidence of the disease is likely to be present from intrauterine life. Rarely, however, kidneys that are anatomically similar may cause death in infancy or early childhood, and the condition has been designated as "adult variety occurring in infancy". ${ }^{2}$ In ADPKD, cysts develop only later in adulthood yet exceptionally cysts may be encountered in utero.

We report a rare case of ADPKD, which was diagnosed by ultrasonography in utero as infantile polycystic kidney disease due to the presence of bilateral enlarged hyperechogenic kidneys with oligohydramnios. Pregnancy was terminated and autopsy revealed it to be an adult polycystic kidney disease. This case is reported due to its rare presentation in utero.

Keywords: Autosomal dominant polycystic kidney disease, Ultrasonography.

\section{INTRODUCTION}

A utosomal dominant polycystic kidney disease is the most widespread cause of genetic nephropathy. Only $25 \%$ of patients are symptomatic. ${ }^{1}$ One in 1,000 people carry the autosomal dominant polycystic kidney disease mutant gene. A utosomal dominant polycystic kidney disease is usually asymptomatic until the third or fourth decade of life, although histological evidence of the disease is likely to be present from intrauterine life. Rarely, however, kidneys that are anatomically similar may cause death in infancy or early childhood, and the condition has been designated as "adult variety occurring in infancy". ${ }^{2}$ In ADPK D, cysts devel op only later in adulthood yet exceptionally cysts may be encountered in utero.

It is a common genetic disorder characterized by progressive renal cyst development, hypertension, and frequently by the development of end stage renal failure. A utosomal dominant polysystic kidney disease (ADPKD) is genetically heterogeneous, the PK D 1 gene has been localized at I6p13.3, ${ }^{3}$ the PKD2 gene at $4 \mathrm{ql} 3-23,{ }^{4}$ and the PKD3 locus remains unknown. PKD1 shows wide phenotypic variability, which includes clinical presentation rarely in utero. The mechanism for such early and severe expression of the disease is at present unknown, but it could include segregation of a modifying gene with inefficient DNA transcription/repair function contributed by the unaffected parent.

\section{CASE REPORT}

A registered patient aged 22 years with 23 weeks of pregnancy came for a regular A NC visit. Her obstetric score was G 2 P1L 1 with married life of 6 years. It was a 2 nd degree consanguineous marriage. The first child was 3-year-old female, alive and heal thy. Her routine investigations were within normal limits. A nomaly scan revealed enlarged, hyperechogenic kidneys (Fig. 1) associated with severe oligohydramnios. There was no associated intrahepatic fibrosis or any other congenital abnormality. B iometric parameters corresponded to 22 weeks of gestation. A provisional diagnosis of infantile polycystic kidney disease was made and a decision to terminate pregnancy was taken in view of incompatibility with life. Pregnancy was terminated with two doses of misoprostol and the fetus was subjected to an autopsy. On external examination, low set ears were present. Gross examination reveal ed enlarged kidneys with cystic changes (Fig. 2).

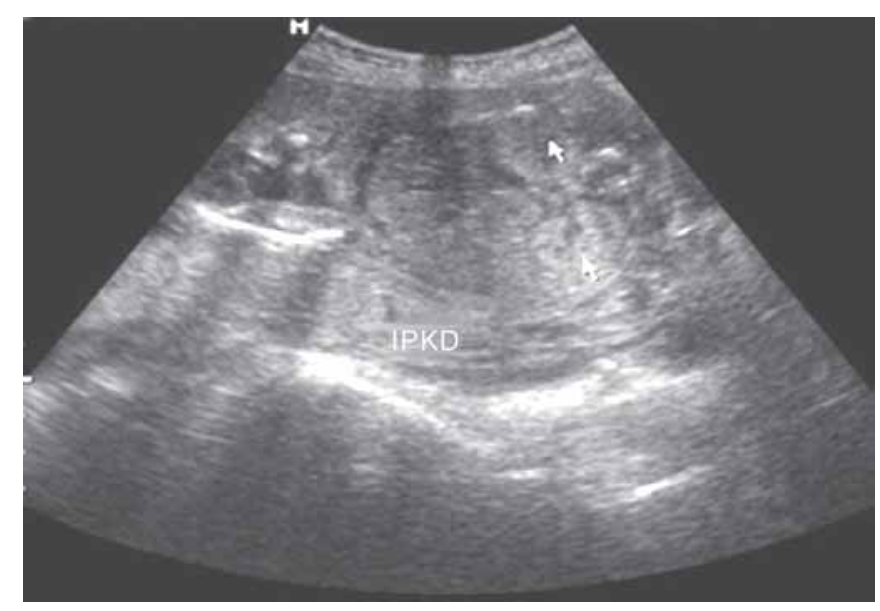

Fig. 1: Ultrasonography film showing bilateral enlarged hyperechogenic kidneys 
Histopathological examination of the kidneys revealed glomerular cystic change suggestive of autosomal dominant kidney disease (Fig. 3). Extramedullary hemopoiesis was seen in liver and spleen.

\section{DISCUSSION}

The occurance of adult polycystic kidney disease in utero is extremely rare. Sonographically various patterns have been described from normal appearance in utero to enlarged and hyperechogenic kidneys with or without multiple cysts. Cysts may develop in utero or after birth in the subcapsular area. This pattern corresponds to gl omerul ocystic variant of disease. The prognosis is still good unless oligohydroamnios develops. In such cases, hypertension and renal failure may develop

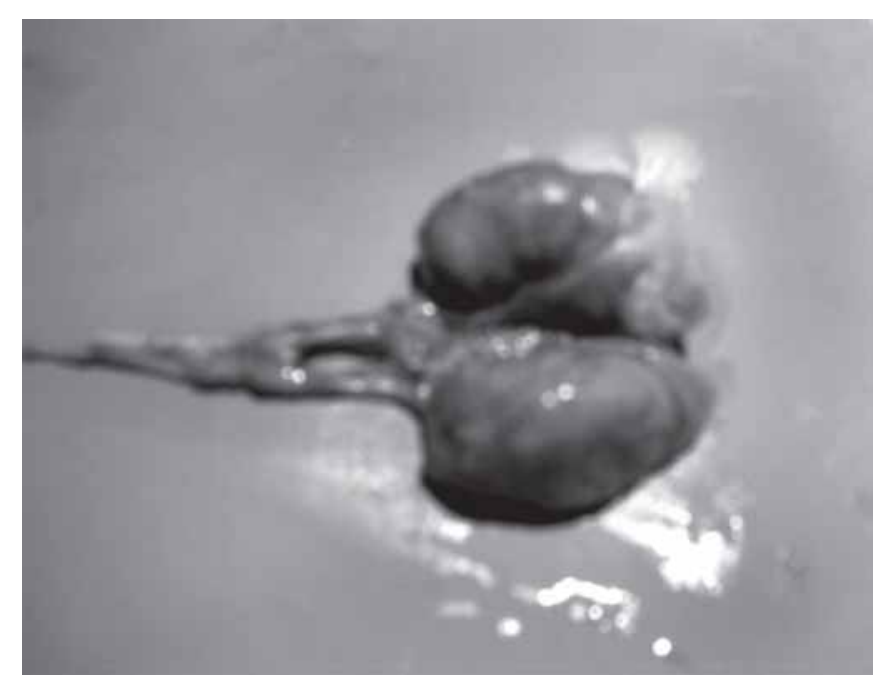

Fig. 2: Macroscopic appearance of bilateral polycystic kidneys and ureters at autopsy

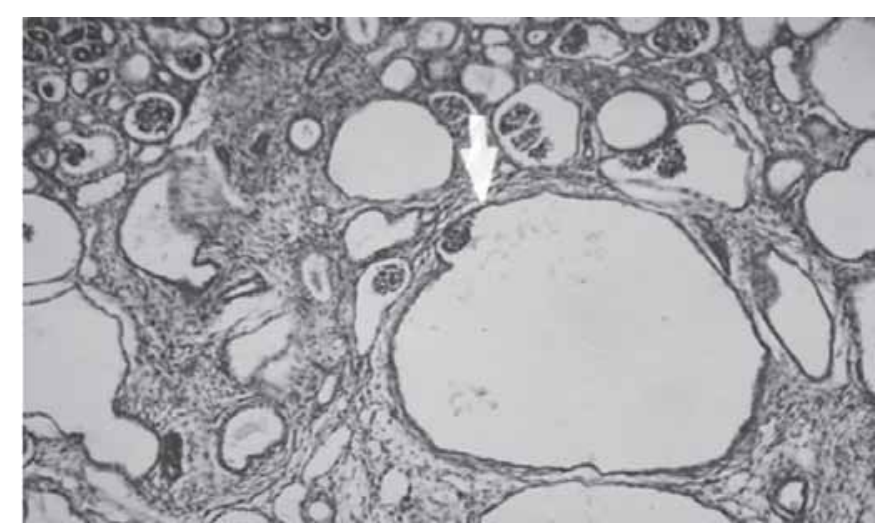

Fig. 3: Microscopic picture showing saccular dilation of collecting tubules, $\mathrm{H} \& \mathrm{E}$ stain rapidly after birth. Other urinary tract malformations may be present in association with A DPKD.

Prenatal diagnosis by ultrasonography is confined to a few case reports and the kidneys have been described as enlarged and hyperechogenic with or without multiple cysts. Unlike infantile polycystic kidneys, where there is a loss of corticomedullary junction in autosomal dominant polycystic kidney disease, there is accentuation of this junction. The amniotic fluid volume is either normal or reduced. The kidney size is usually smaller than the infant polycystic kidney. Autosomal dominant polycystic kidney disease in the fetus should be suspected when cystic enlarged kidneys are detected in association with a normal amount of amniotic fluid. The anomaly can be found as late as the third trimester with normally looking kidneys in midtrimester. ${ }^{5} \mathrm{~T}$ he diagnosis of intrauterine or neonatal autosomal dominant polycystic kiney disease should prompt investigation of both parents. Molecular analysis of autosomal dominant polycystic kidney disease allows presymptomatic diagnosis in individuals younger than age 30 and helps in establishing prognosis. ${ }^{6}$

If the diagnosis is made before viability, the option of pregnancy termination should be offered to the parents. A fter viability, the diagnosis of autosomal dominant polycystic kidney disease probably should not alter standard obstetrical management. If any time enlarged hyperechogenic kidneys are diagnosed in a fetus, members of the family should be screened with renal sonography.

\section{REFERENCES}

1. Serbelloni P, Conte F, Garavaglia G, Spattini A, Sessa A. Echographic screening for autosomal dominant polycystic kidney disease from intrauterine life to adult life. A rch Ital U rol Nefrol Androl J un 1991;63 (Suppl 2):89-92.

2. Pilu G, Nicolaides KH. Diagnosis of fetal abnormalities: The 18-23 week scan. Diploma in fetal medicine series. The Parthenon Publishing Group, New Y ork/L ondon 1999;80-81.

3. Reeders ST, B reuning MH, Davies KE, et al. A highly polymorphic DNA marker linked to adult polycystic kidney disease on chromosome 16. N ature 1985;317:542-44.

4. K imberling W I, K umar S, Gabow PA, et al. A utosomal dominant polycystic kidney disease: L ocalization of the second gene to chromosome 4ql3-q23. Genomics 1993;18:467-72.

5. M ain D, M ennuti M T, Cornfeld D. Prenatal diagnosis of adult polcystic kidney disease. Lancet 1983;2:337.

6. Torra R, B adenas C, Darnell A, Nicolau C, V olpini V, Revert L, Estivill $X$. L inkage, clinical features, and prognosis of autosomal dominant polycystic kidney disease types 1 and 2 . J A m Soc Nephrol Oct 1996;7(10):2142-51. 